\title{
Comparison of RFIC PA Die and Laminate Co-Simulation Methods
}

\author{
Liu Xilei ${ }^{1,2, *}$, Dai Dajie², Chen Sidi $^{2}$, Yang Hanbing ${ }^{2}$, Li Xuejian², Zhou Xianhua ${ }^{2}$, Yue Bingdi ${ }^{2}$ \\ ${ }^{1}$ School of Cultural Creativity and Tourism, Guangdong University of Finance \& Economics, Guangzhou, China \\ ${ }^{2}$ Guangzhou Runxin Information Technology Co. Ltd, Guangzhou, China
}

Email address:

xileiliu@gdufe.edu.cn (Liu Xilei)

${ }^{*}$ Corresponding author

\section{To cite this article:}

Liu Xilei, Dai Dajie, Chen Sidi, Yang Hanbing, Li Xuejian, Zhou Xianhua, Yue Bingdi. Comparison of RFIC PA Die and Laminate Co-Simulation Methods. Journal of Electrical and Electronic Engineering. Vol. 6, No. 4, 2018, pp. 111-119. doi: 10.11648/j.jeee.20180604.12

Received: September 18, 2018; Accepted: September 30, 2018; Published: October 29, 2018

\begin{abstract}
Package (such as LGA, MCM etc.) with main circuits on Die(s) situated on a substrate and with OMN in the substrate laminate is one of the most popular design styles for RFIC PAs in recent times. In practical design, the overall circuit of the RFIC PA is often designed using ADS Schematic simulation tool. The main circuit is then layout for Die design, and the OMN circuit is layout for laminate design. As the actual parasitics associated with the real RF circuits with high packing dense have significant effects on the RFIC performance, the traditional closed-form circuit models may be no longer valid, and co-simulations of the Die circuit or Die layout EM model and the laminate layout EM model are often considered in order to improve simulation accuracy. There are some generally considered co-simulation methods in practical design, such as Die circuit + Laminate Momentum, Die circuit +Laminate HFSS, Die layout Momentum +Laminate Momentum, Die layout Momentum +Laminate HFSS, Die layout +Laminate ADS Nested Technology, etc. Design engineers might be confused about which co-simulation methods should take for RFIC PA design and development. This paper reviews, compares and discusses the above co-simulation methods in the aspects of algorithms of the simulators, co-simulation modeling method, complexity to build model, run time, and the difference between simulation and measurement results by giving an example of a self-developed RFIC PA operating at $2 \mathrm{GHz}$. It endeavors to draw helpful conclusions for simulation experiences in practical RFIC PA design, and a co-simulation method with Die layout Momentum EM model +Laminate HFSS EM model is recommended based on the software versions used in this work.
\end{abstract}

Keywords: Microelectronics Technology, RFIC PA, Die and Laminate Co-simulation Method, Comparison of Momentum, HFSS and ADS Nested technology

\section{Introduction}

Package (such as LGA, MCM etc.) with main circuits on Die(s) situated on a substrate and with Output Matching Network $(\mathrm{OMN})$ in the substrate laminate is one of the most popular design styles for Radio Frequency Integrated Circuit Power Amplifier (RFIC PA) in recent times [1-3]. In practical design, the overall circuit of the RFIC PA is often designed using ADS Schematic simulation tool, for ADS provides a best-in-class harmonic balance simulator for RF simulations [4]. The main circuit is then layout for Die design, and the $\mathrm{OMN}$ circuit is layout for laminate design. As the actual parasitics associated with the real RF circuits with high packing dense have significant effects on the RFIC performance, the traditional closed-form circuit models may be no longer valid, and co-simulations of the Die circuit or layout Electromagnetics (EM) model and the laminate layout EM model are often considered in order to improve simulation accuracy [5-7]. There are some generally considered co-simulation methods [5-10] in practical design, such as Die circuit + Laminate Momentum, Die circuit +Laminate HFSS, Die layout Momentum +Laminate Momentum, Die layout Momentum +Laminate HFSS, Die layout +Laminate ADS Nested Technology, etc. Design 
engineers might be confused about which co-simulation methods should take for RFIC PA design and development. This paper reviews, compares and discusses the above co-simulation methods in the aspects of algorithms of the simulators, co-simulation modeling method, complexity to build model, run time, and the difference between simulation and measurement results by giving an example of a self-developed RFIC PA operating at $2 \mathrm{GHz}$ by tools ADS2014 and HFSS15.0. It endeavors to draw helpful conclusions for simulation experiences in practical RFIC PA design.

The Top level of the schematic hireachy of the example RFIC PA (PA\#L03) in this paper is shown in Figure 1
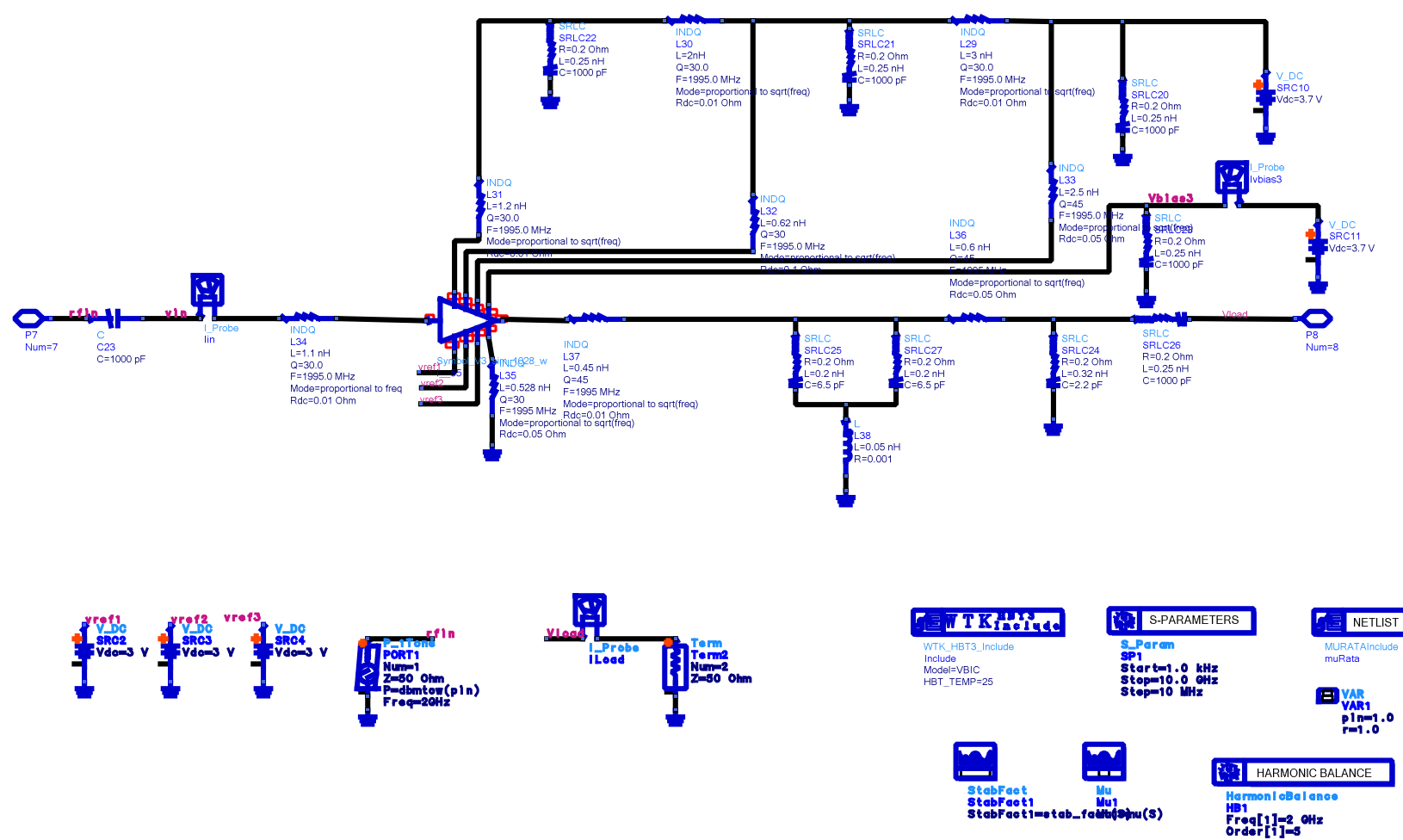

Figure 1. ADS Schematic Design of this Example.

\section{Algorithms of Various Simulators}

ADS is a commercial simulation tool for RFIC design, developed by Agilent Inc. There are two kinds of EM simulators in ADS: Momentum Microwave and FEM. The Momentum algorithm is based on MoM (method of moment), and FEM is based on a finite element method. HFSS is a high frequency EM 3D full wave simulation software product of Ansys Ansoft Inc., based on FEM. The MoM and FEM are summarized as follows $[1,2]$.

\subsection{FEM}

The finite element method (FEM) is proposed in 1940s, then has been widely used in structural analysis. FEM is a numerical method based on variation rountine. By applying variation routine, the boundary problems are transformed into variation problems. By mesh, interpolation of the region, the variation problems are discretized into extremum problem of common multivariate functions, then multivariant algebraic equations are obtained. The boundary problems are then solved by solving these equations. The processes are usually as follows:

i. Mesh region. Mesh the region or object into finite elements, such as triangle, rectangle, cubic, hexahedron etc.;

ii. Select interpolation functions. Select the style of the Interpolation functions such as polynomial, then solve the appromative field value of any subregion through the field value of the node. Normally the higher order of the interpolation function, the more complex of the formulas.

iii. Build the equations. It can be built by Ritz method or Galerkin method.

iv. Solve the equations in appropriate methods.

\subsection{MoM}

The method of moments (MoM) is one of the most commonly used methods in computational electromagnetics. Since Harrington put forward the basic concept of moment method in the 1960s, it has been widely used in engineering, especially in the fields of electromagnetic radiation, scattering and electromagnetic compatibility.

The basic idea of MoM is to separate the geometric object, define the proper basis function on it, then establish the integral equation, test the weight function to produce a matrix equation, solve the matrix equation, then get the current distribution on 
the geometric object, so that other near and far field information can be obtained from the current distribution.

The basic solving processes of MoM are described as follows:

i. Discretization Process -to transform operator equations into algebraic equations.

A set of linearly independent basis functions (or expansion functions) $f_{1}, f_{2}, \cdots, f_{n}$ is appropriately selected for the definition domain of operators $L$ in operator equations $L(f)=g$. The unknown function $f$ is expanded into a linear combination of basis functions in the definition domain of operators $L$, and the finite term approximation is adopted, ie. $f=\sum_{n=1}^{\infty} a_{n} f_{n} \approx f_{N}=\sum_{n=1}^{N} a_{n} f_{n}$. Then this equation is substituted into the operator equation, and the operator equation is transformed into an algebraic equation, ie. $\sum_{n=1}^{N} a_{n} L\left(f_{n}\right)=g$ by using the linear property of the operator. Therefore, the problem of solving unknown functions $f$ is transformed into the problem of solving coefficients $a_{n}$.

ii. Sampling Test Process - In order to minimize theerror between the approximate function $f_{N}$ of the unknown function $f$, a sampling test must be performed to determine the unknown coefficient $a_{n}$ by making the weighted average error zero at the sampling point.
A set of linearly independent weighting functions (also called test functions) $W_{m}$ are appropriately selected in the range of the operator $L$ and the inner product of the above algebraic equation is taken for sampling test, i.e. $\left\langle L\left(f_{n}\right), W_{m}\right\rangle=\left\langle g, W_{m}\right\rangle \quad(m=1,2, \cdots, N)$. By using the linear and inner product properties of operators, it is transformed into matrix equation $\sum_{n=1}^{N} a_{n}\left\langle L\left(f_{n}\right), W_{m}\right\rangle=\left\langle g, W_{m}\right\rangle \quad(m=1,2, \cdots, N)$ So the problem of solving algebraic equation is transformed into solving matrix equation.

iii. Matrix Inverse Process - Once the matrix equation is obtained, the solution of the matrix equation can be obtained by using the conventional matrix inversion or solving the linear equations, and the expansion coefficients $a_{n}$ can be determined, then the solution of the original operator equation can be obtained. According to the above algorithm principle, Table-1 summarizes and compares the two algorithms performances of MoM and FEM.

As for the FEM simulator with ADS and HFSS, their algorithm principles might be the same, but the specific implementation approaches of the algorithm engine program and the setting of boundary conditions may be different [13, 14]. In addition, ADS can simulate active devices while HFSS is not capable.

Table 1. MoM VS FEM.

\begin{tabular}{|c|c|c|}
\hline Performance & МoM & FEM \\
\hline Solutions & Modelling of antenna, strip \&planarlwiring structures & $\begin{array}{l}\text { Electrical properties and physical entities can be defined } \\
\text { and processed separately }\end{array}$ \\
\hline Numerical Modelling Merits & $\begin{array}{l}\text { Stable; "pseudo-solution" not easy to appear; good matrix } \\
\text { conditions easy to solve and inverse; but with dense matrix } \\
\text { leading to mass data storage and computational complexity }\end{array}$ & $\begin{array}{l}\text { The resulting linear algebraic equations are generally } \\
\text { correlated to positive definite sparse coefficient matrices }\end{array}$ \\
\hline EM Field Solving Region & E\&H Field at any location outside the radiator & $\begin{array}{l}\text { Suit for objects with complicated shapes and boundary } \\
\text { problems; Efficient for inner region EM problem }\end{array}$ \\
\hline Applications & $\begin{array}{l}\text { Calculate antenna parameters, input impedance, gain, radar } \\
\text { problems etc. }\end{array}$ & $\begin{array}{l}\text { convenient to handle multiple medium and non-uniform } \\
\text { continuous medium problems }\end{array}$ \\
\hline $\begin{array}{l}\text { Difficulties in Numerical } \\
\text { Modelling }\end{array}$ & Difficulties in modeling inner region & $\begin{array}{l}\text { Must model the no boundary problem as a boundary } \\
\text { problem }\end{array}$ \\
\hline Computer Implementation & $\begin{array}{l}\text { Difficulties in solving non-uniform medium; large resources } \\
\text { consumption;low-frequency applications only. }\end{array}$ & $\begin{array}{l}\text { Intensive computation; difficult to handle unknown field } \\
\text { problems on closed surfaces in open sites. }\end{array}$ \\
\hline
\end{tabular}

\section{Comparison of Co-Sim Methods}

This paper reviews and compares several co-simulation methods of RF PA Die and substrate laminate drawn from the practical engineering experiences. The manuals of the related softwares may be referred to $[3,4$,$] and other relevant training$ materials from web:

\subsection{Method 1: Die Circuit + Laminate Momentum}

Step 1:ADS ports are set up where the designed PA laminate layout hook the active device, passive device and external power supply, as shown in Figure. 2.a). The substrate EM file (Subst) is defined as in Figure 2.b).

Step2: While setting up the ADS EM simulation, create Symbol and select the Symbol type as 'Layout look-like', select 'Momentum Microwave' and run EM simulation. emModel Symbol will be updated automatically after the simulation.

Step3: In ADS schematic cell, the PA laminate layout Symbol simulated in step 2 is hooked to the PA bare Die circuit packed in Symbol, as shown in Figure. 3. 


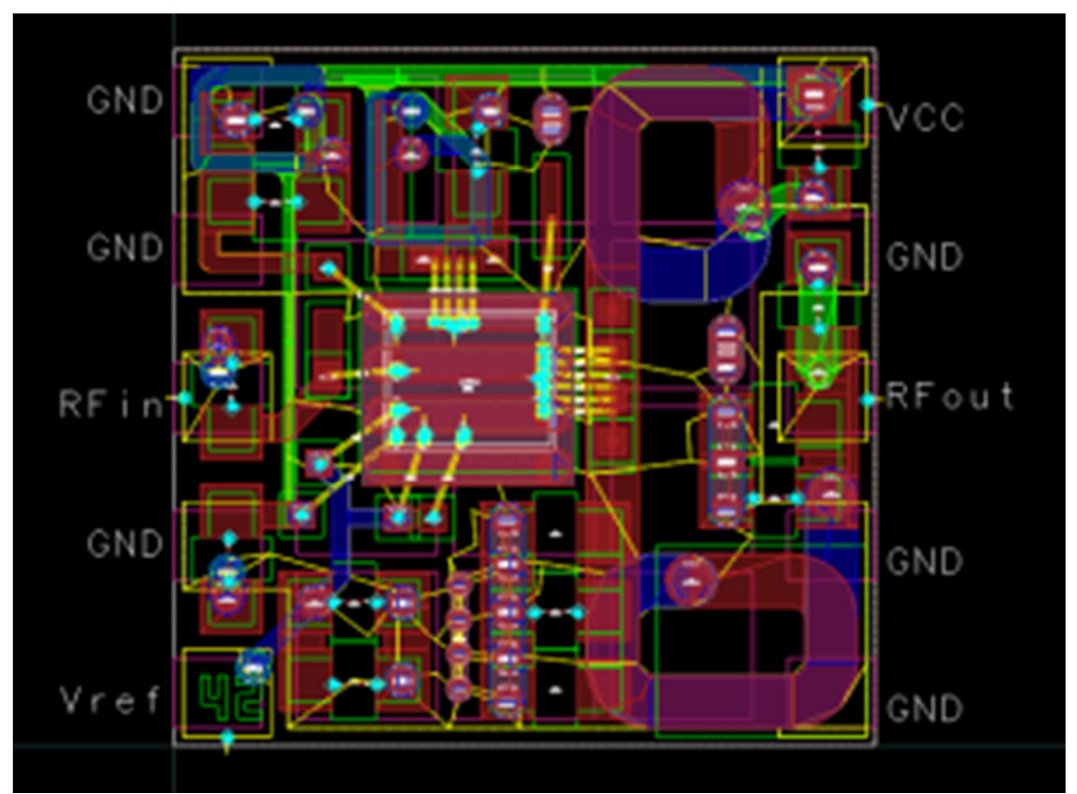

a) EM port setup for laminate

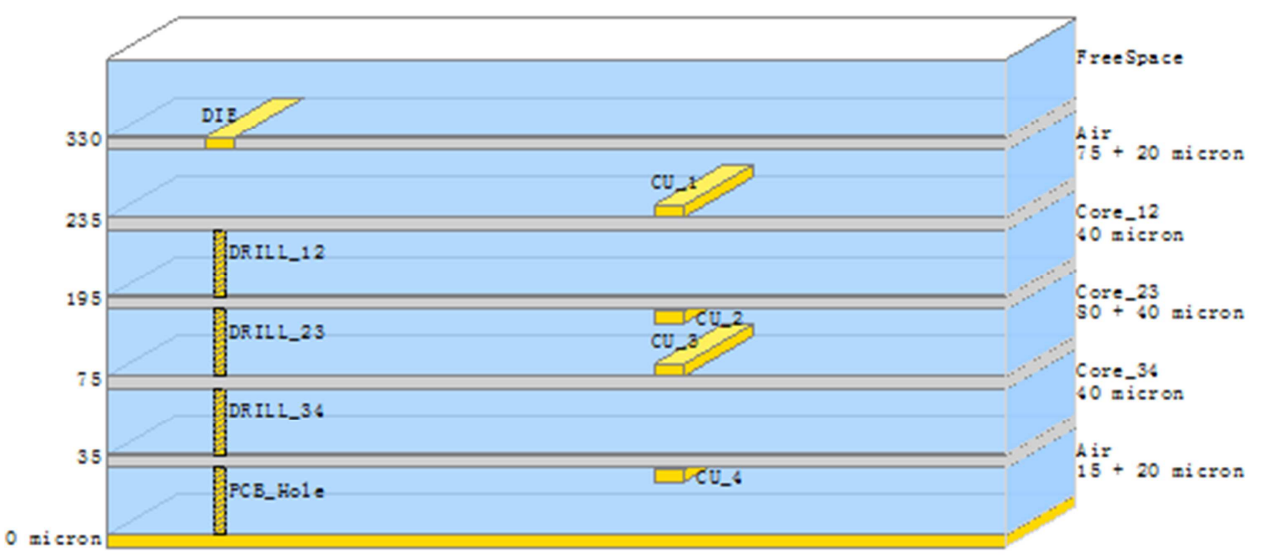

b) EM substrate setup for laminate

Figure 2. Laminate momentum EM port and substrate setup.

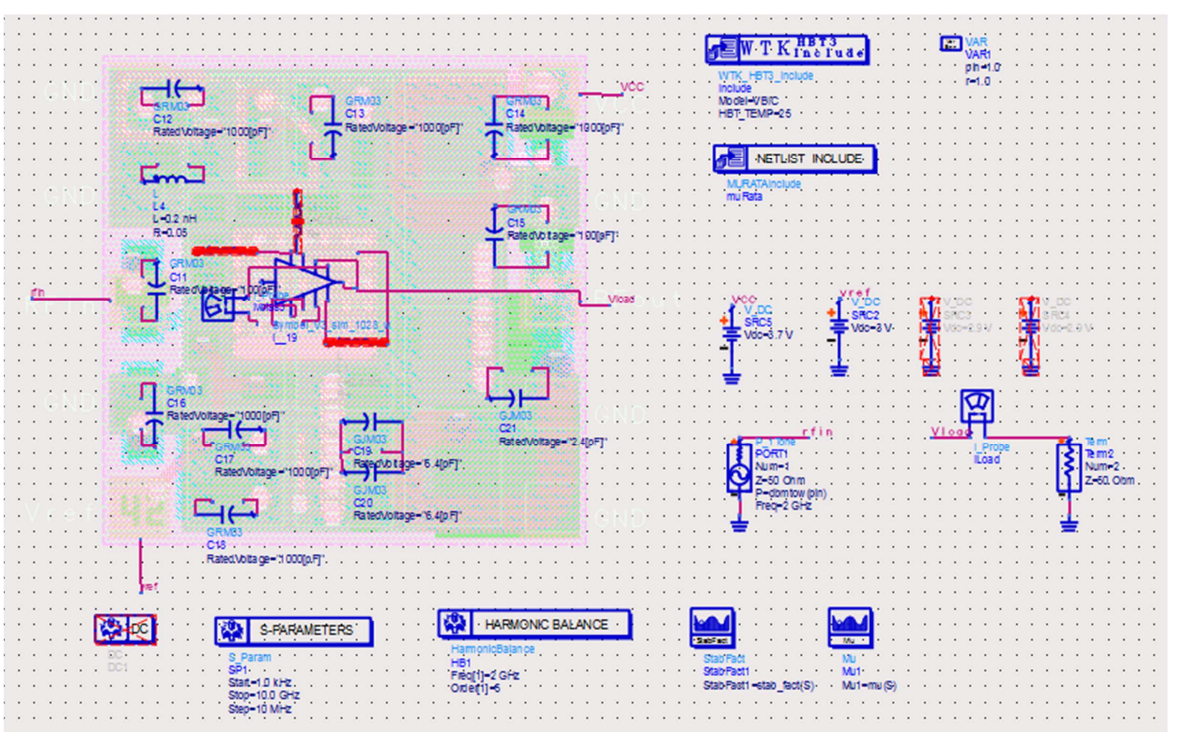

Figure 3. Co-sim schematic setup of Die circuit + laminate Momentum. 
Step4: Run PA simulations of the co-sim circuit set up in Step3.

\subsection{Method2: Die Circuit+ Laminate HFSS (Lumpedport No Deembed)}

Step 1: The PA laminate layout from ADS is import into HFSS. HFSS lumpedports are set where the active device, passive device and external power supply are hooked, as shown in Figure 4. The 'Deembed' Option of Lumpedport set up is not set in Method 2.

Step 2: Run HFSS simulation and export the $\mathrm{N}$ port $\mathrm{S}$ parameter file (SNP) after the simulation.

Step 3: In ADS schematic cell, hook the PA substrate laminate layout S parameter file (SNP) exported from Step 2 to the PA bare Die circuit packed in Symbol, as shown in Figure. 5.

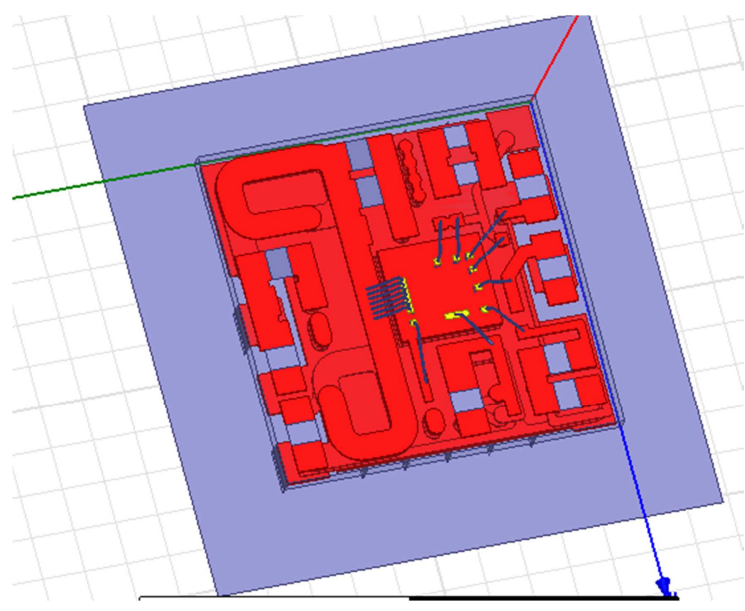

Figure 4. Laminate Simulation Setup in HFSS.

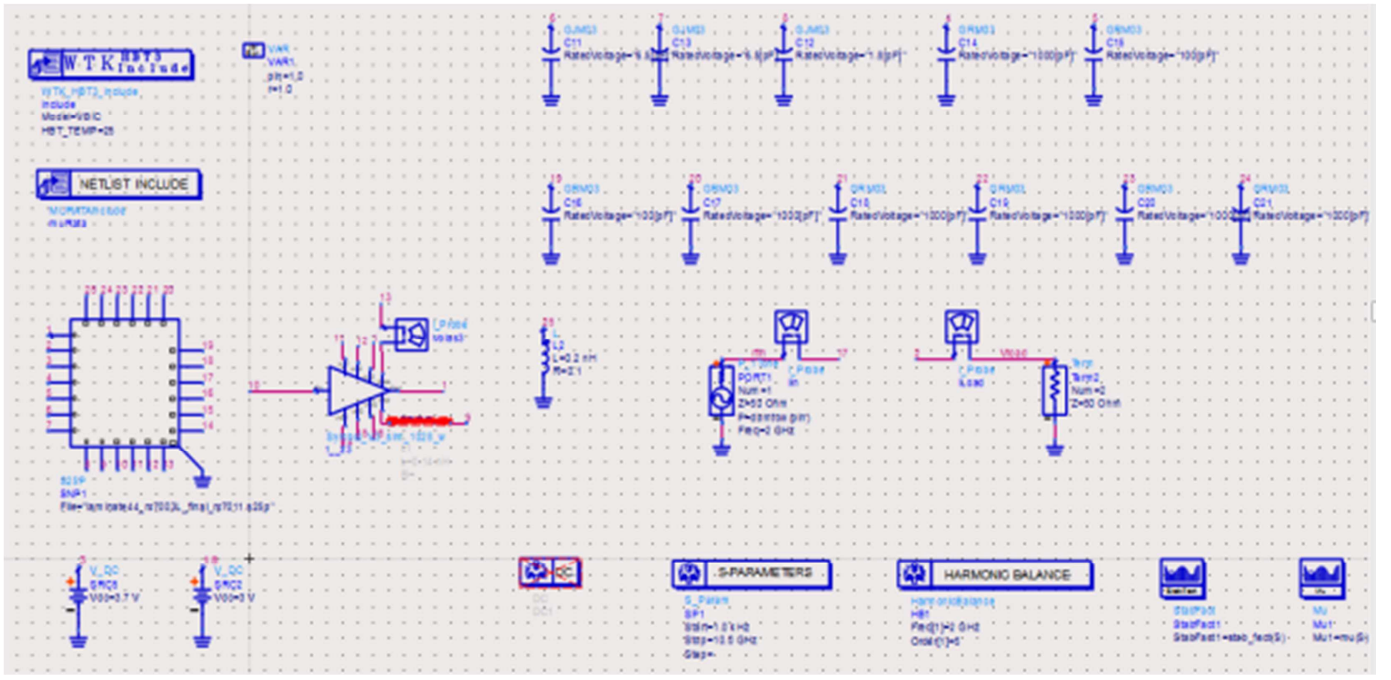

Figure 5. Co-sim schematic setup of Die circuit + laminate HFSS.

\subsection{Method 3: Die Circuit + Laminate HFSS (Lumpedport Deembed)}

Steps in Method 3 are as that in Method2, except that the lumpedport setup in HFSS is set as 'Deembed'.

\subsection{Method 4: Die Layout Momentum + Laminate HFSS}

Step 1: Import the PA Die layout (from Cadence Virtuoso in this example) into ADS layout.

Step2:For EM simulation of complete PA Die layout, due to the large number of ports required, the resources and memory consumption for simulation are also very large. At present, the simulation takes very long running time, or even impossible to run with a moderate machine environment. Method 4 usually needs to simplify the PA Die layout. Since high frequency parasitics mainly affect the output stage of PA, all the active devices and resistors are removed and only the output stage and pads are retained in PA Die layout in Method 4. Then similar to Step 1 in Method 1, the shortcut layout is configured with ADS ports, and three PORTs are set for each electrode of the triode, as shown in Figure 6.
Step 3: Similar to Method 1 Step 2, create Symbol and select the Symbol type as 'Layout look-like' in ADS, select 'Momentum Microwave' and run EM simulation. emModel of Die layout will be automatically updated after the simulation.

Step 4: Simulate PA substrate laminate and export.SNP file from HFSS as instructed in Method 2 Step1-2.

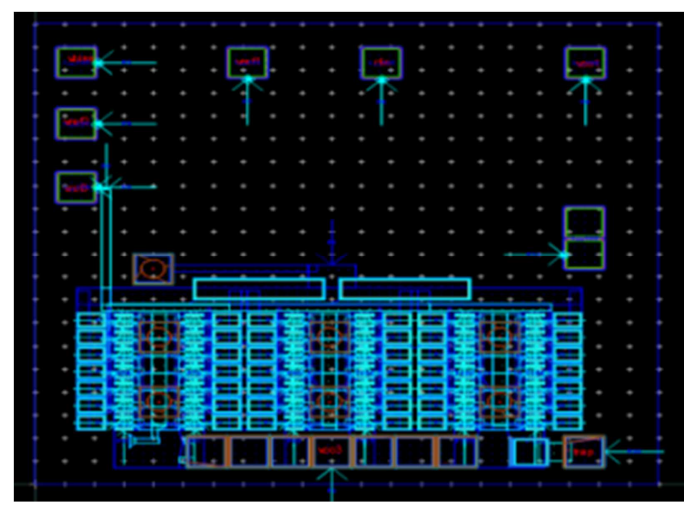

Figure 6. Shortcut PA Die layout and its ADS Momentum port setup. 
Step 5: In ADS schematic cell, hook the PA Die layout emModel simulated in Step1-3 and the.SNP file in Step 4.

Step 6: Run co-simulation of the configuration in Step 5.

\subsection{Method5: Die Layout Momentum +Laminate Momentum}

Step 1: Similar to Method 4 Step1-3, simulate the PA Die layout by Momentum, and obtain the Die layout emModel.

Step 2: Similar to Method 1 Step1-3, simulate the PA substrate laminate layout by Momentum, and obtain the laminate emModel.

Step 3: In ADS schematic cell, hook the PA Die layout emModel, the missing active and passive devices and the laminate emModel, partially shown as Figure 7

Step 4: Run co-simulation of the configuration in Step 3.

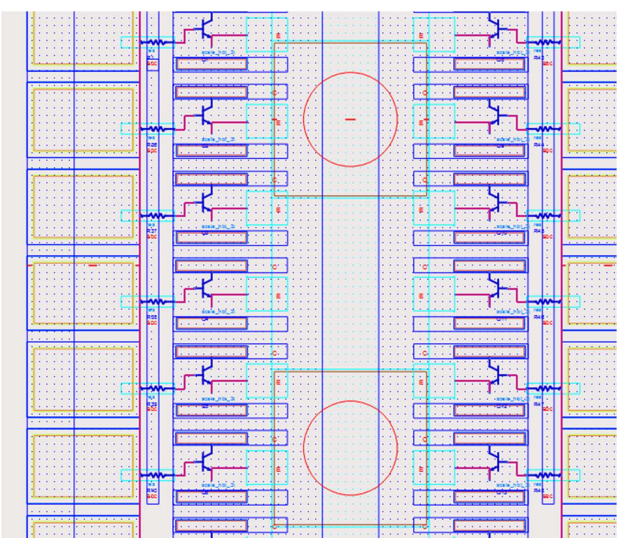

Figure 7. PA Die Layout emModel hooking active, passive devices (part).

\subsection{Method6: Die Layout+ Laminate ADS Nested Technology}

See ADS Help for manual of ADS Nested Technology. The nested configuration are shown as Figure 8.

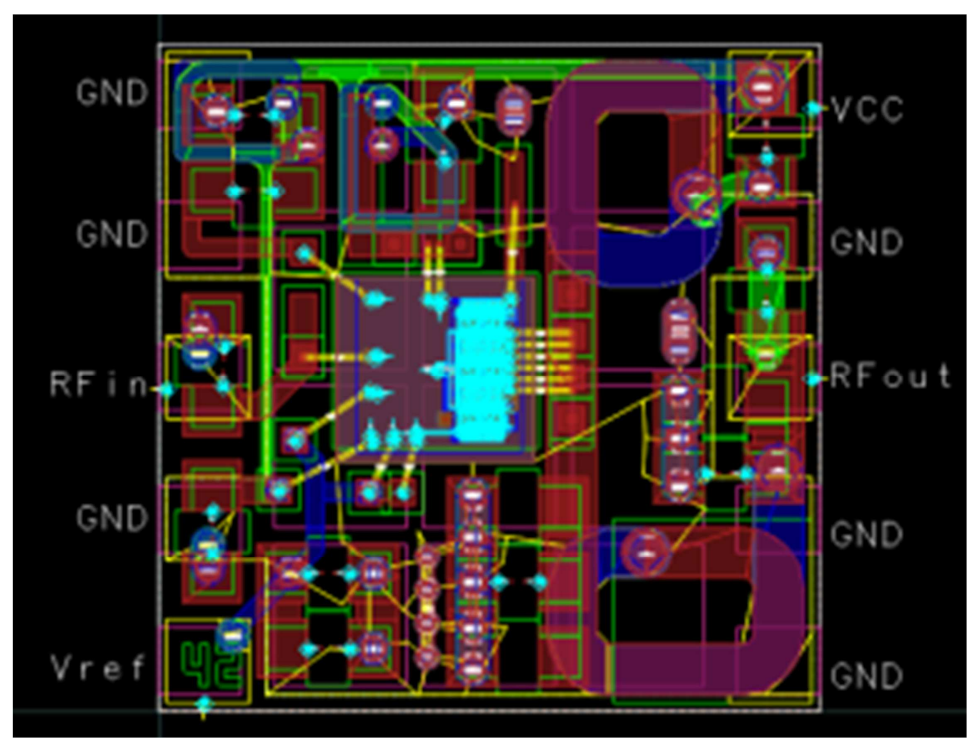

a) Port setup

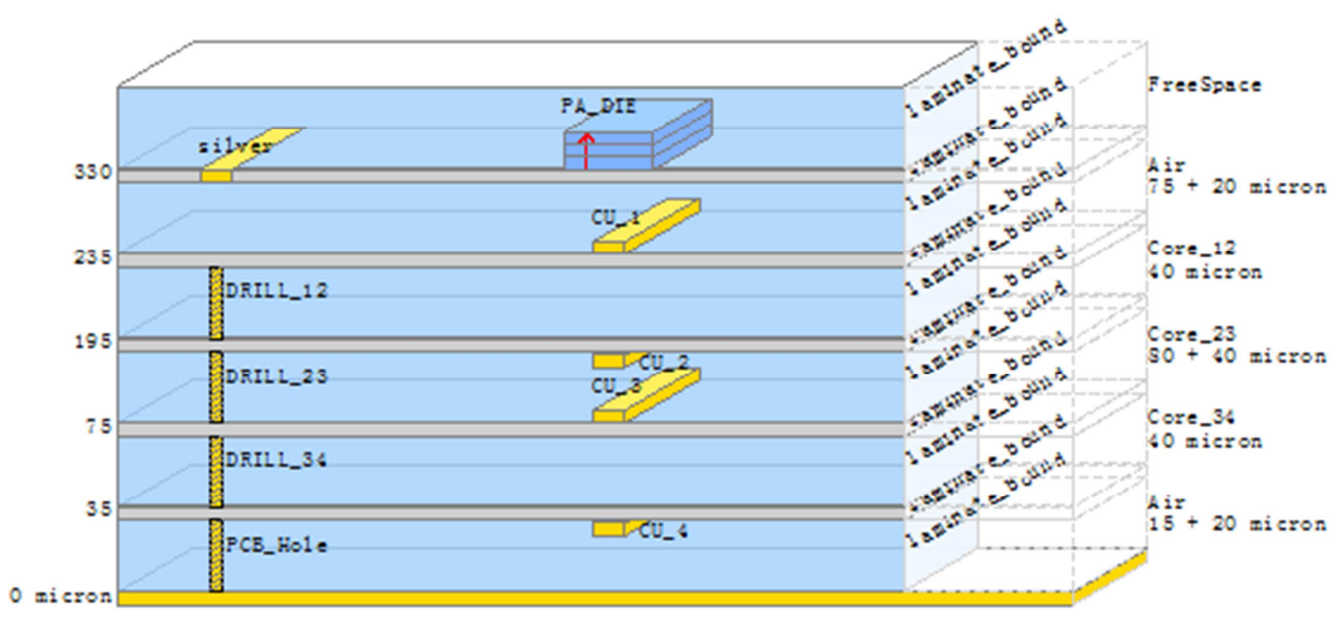

b) Die on laminate Nested substrate EM setup

Figure 8. ADS Nested Technology configuration setup of this example. 
In this example, the chip (PA\#L03) are simulated by all the methods described above. The complexity to build model, run time of these methods are compared as shown in Table -2. (Running System: 2Core 3.2GHz i5 CPU, 16GB RAM, 64-bit; Software Version: ADS 2014, HFSS 15.0):

Table 2. Comparisons of Model complexity and Run time.

\begin{tabular}{|c|c|c|c|c|}
\hline Method No. & Description & $\begin{array}{l}\text { EM Ports } \\
\text { Count }\end{array}$ & $\begin{array}{l}\text { Complexity to build model } \\
\text { (Score 1 6, less the easier) }\end{array}$ & Run Time \\
\hline M1 & Die Circuit scheme + Laminate Momentum & 35 & 1 & $\sim 4 \mathrm{hrs}$ \\
\hline M2 & Die Circuit scheme + Laminate HFSS (Lumpedports No Deembed) & 25 & 2 & $\sim 45 \mathrm{~min}$ \\
\hline M3 & Die Circuit scheme + Laminate HFSS (Lumpedports Deembed) & 25 & 2 & $\sim 45 \mathrm{~min}$ \\
\hline M4 & Die LayoutMomentum + Laminate HFSS & $138+25$ & 5 & $\sim 3.5 \mathrm{hrs}+\sim 45 \mathrm{~min}$ \\
\hline M5 & Die Layout Momentum+ Laminate Momentum & $138+35$ & 4 & $\sim 3.5 \mathrm{hrs}+\sim 4 \mathrm{hrs}$ \\
\hline M6 & Die Layout +Laminate ADS Nested Technology & 163 & 6 & $\sim 15 \mathrm{hrs}$ \\
\hline
\end{tabular}

\section{Simulations VS Measurements}

The above example PA\#L03 chip has been taped out and conducted DOE measurements. The simulation results and the measurements results are compared, as shown in Table-3 (C11, $\mathrm{C} 12$ are capacitors of the first stage of OMN respectively, and
C21 is capacitor of the second stage of OMN; Pout is output power; Pin is input power; PAE is power additional efficiency; ICQ is quiescent current; $\mathrm{G}$ is gain; $\mathrm{S}$ is ADS simulation of circuit schematic only; $\mathrm{T}$ is testing).

Table 3. Simulations VS Measurements.

\begin{tabular}{|c|c|c|c|c|c|c|c|c|c|c|}
\hline \multicolumn{11}{|c|}{ @VCC $=3.7 \mathrm{~V}$, Vref=3V, Freq $=2 \mathrm{GHz}$, Pin=10dBm or Pout $=$ Psat } \\
\hline \multirow{3}{*}{ Method } & \multirow{3}{*}{$\begin{array}{l}\text { ICQ } \\
(\mathrm{mA})\end{array}$} & \multicolumn{3}{|c|}{ @OMN 1} & \multicolumn{3}{|l|}{ @OMN 2} & \multicolumn{3}{|l|}{ (a) OMN 3} \\
\hline & & C11: 6.5pF & C12: 6.5pF & C21: 2.2pF & C11: 6.4pF & C12: 6.4pF & C21: 2.4pF & C11: 6.0pF & C12: 6.0pF & C21: 2.0pF \\
\hline & & Pout (dBm) & PAE (\%) & G@Pin & Pout (dBm) & PAE (\%) & G@Pin & Pout (dBm) & PAE (\%) & G@Pin \\
\hline $\mathrm{S}$ & 258 & 32.5 & 38.9 & 22.5 & 32.8 & 41.5 & 22.8 & 34 & 44.7 & 24 \\
\hline $\mathrm{T}$ & 333 & 33.7 & 33.2 & 23.7 & 33.9 & 36.7 & 23.9 & 33.2 & 29.8 & 23 \\
\hline M1 & 258 & 35 & 44.1 & 25 & 34.4 & 40.4 & 24.4 & 33.8 & 37 & 23.8 \\
\hline M2 & 258 & 34.4 & 44 & 24.4 & 34.6 & 42.7 & 24.6 & 34.8 & 45 & 24.8 \\
\hline M3 & 258 & 35 & 47 & 25 & 35.6 & 49.1 & 25.6 & 35.6 & 53.4 & 25.6 \\
\hline M4 & 253 & 34.2 & 45.8 & 24.2 & 35 & 51.8 & 25 & 35.2 & 55.5 & 25.2 \\
\hline M5 & 257 & 29.2 & 37.7 & 23.2 & 29.2 & 42.5 & 23.2 & 30.1 & 46.1 & 24.1 \\
\hline M6 & 257 & 29.8 & 43.6 & 25.8 & 31.1 & 36.1 & 27.1 & 32 & 40.5 & 28 \\
\hline
\end{tabular}

More straightforward comparisons are illustrated in Figure 9.

\section{Discussions}

The above sections reviews and compares several generally considered co-simulation methods of Die and laminate in practical RFIC PA design in the aspects of algorithms of the simulators, co-simulation modeling methods, complexity to build model, run time, and the difference between simulation and measurement results by giving an example of a self-developed RFIC PA. It endeavors to draw the following conclusions that might be helpful for simulation experiences in practical RF PA IC design (based on the software versions used in this work) in this section:

i. The results of ADS simulation with circuit schematic only (Method S) are close to the measured ones and quick, but in order to improve the simulation accuracy, it is suggested to carry out the EM co-simulation of substrate laminate.

ii. The method of co- simulation with Die circuit Schematic and substrate laminate EM simulation by HFSS with lumpedports no deembed setting (Method 2) is closer to the measured results than that with deembed lumpedport setting (Method 3). The publication [17] presents discovery of intrinsic port inductance in an
HFSS model and discusses issues and techniques on how to handle lumped SMT ports in an HFSS EM model for more accurate SMD circuit simulation. Further investigations in [17] may give a more accurate HFSS EM model of the laminate layout.

iii. The method of co- simulation with Die circuit schematic and substrate laminate EM simulation(Method 1,2) is more close to the measured results than ADS simulation with circuit schematic only (Method S): the difference of quiescent current is about $22 \%$; difference of the output power is about $0.8-1.6 \mathrm{~dB}$; and the efficiency difference is about $11-33 \%$. From view of accuracy, there is little difference between Method 1 and Method 2 , but the simulation time of HFSS(Method 2) is much less than that of Momentum (Method 1), which is understandable from the principles of FEM and MoM algorithms.

iv. The method of co-simulation of Die layout emModel (by Momentum) and substrate laminate emModel (by HFSS) (Method 4) is closer to the measurement inPout and G thanMethod 1 and Method 2, but it takes longer time and the simulation of efficiency is not accurate enough.

v. The method of co-simulation of Die layout emModel (by Momentum) and substrate laminate emModel (by 
Momentum) (Method 5) is not accurate and time-consuming, and is not recommended.

vi. Using ADS Nesting Technology (Method 6) is inaccurate and time-consuming and is not recommended too.
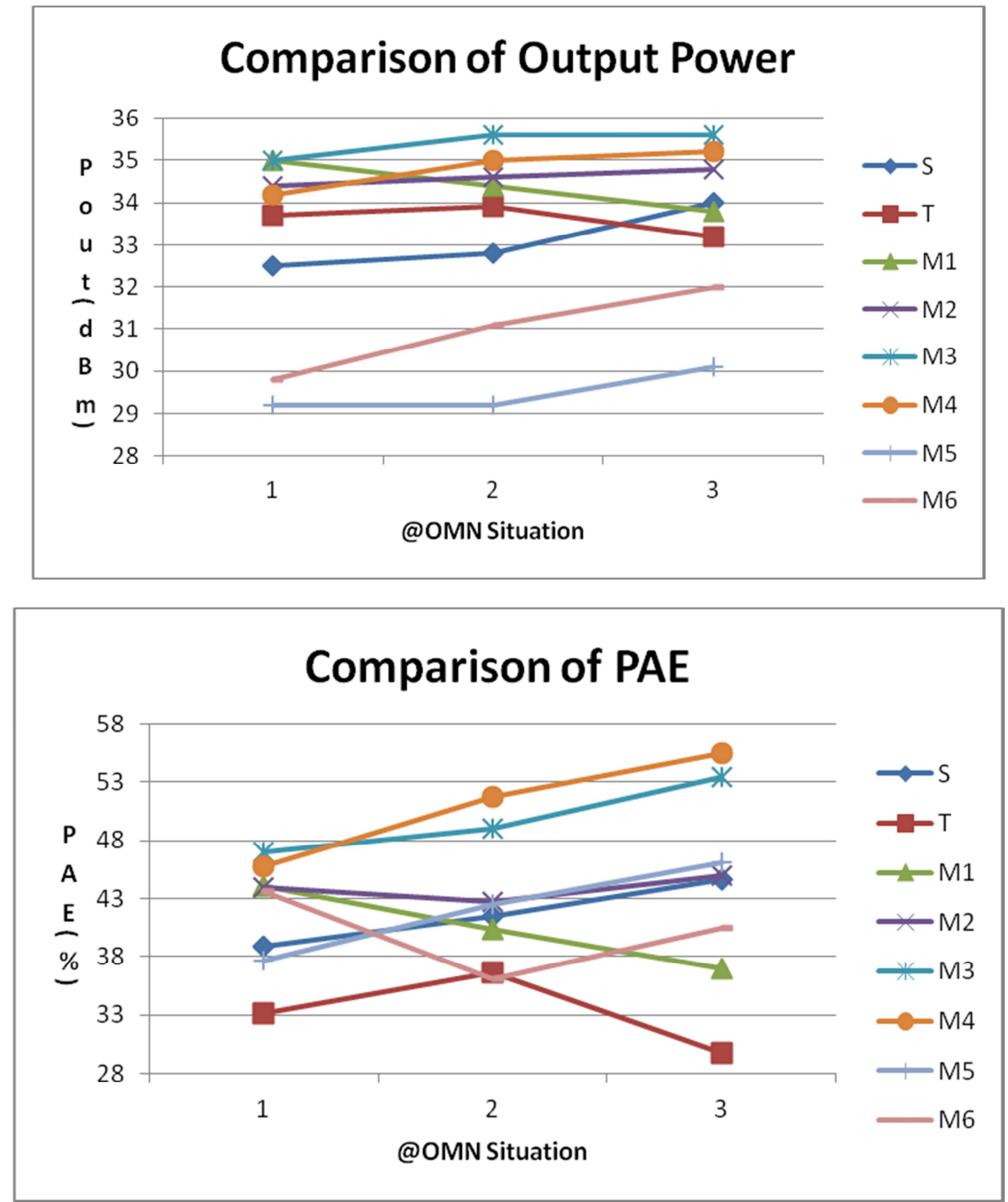

Figure 9. Illustration of Table-3.

\section{Conclusions}

This paper reviews, compares and discusses several generally considered co-simulation methods of Die and laminate in practical RFIC PA design in the aspects of algorithms of the simulators, co-simulation modeling methods, complexity to build model, run time, and the difference between simulation and measurement results by giving an example of a self-developed RFIC PA. It endeavors to draw helpful conclusions for simulation experiences in practical RFIC PA design as stated in Section 5, and a co-simulation method with Die layout Momentum EM model +Laminate HFSS EM model with further considerations on lumped SMT ports handling is recommended based on the software versions used in this work.

\section{References}

[1] Chen Sidi, Zheng Yaohua, and Zhang Guohao. Design of a highly efficient and high harmonic suppression power amplifier [J]. Microelectronic Technology, 2015, vol. 41, No. 4:60-68.

[2] Skyworks-Product-Datasheets -Amplifiers Category, Up to date (2018) available from website: http://www.skyworksinc.com

[3] Quovo-Product-Datasheets-3G/4G Mobile Power Amplifier Category, Up to date(2018) available from website: http://www.qorvo.com

[4] B Wan, X Wang. Overview of commercially-available analog/RF simulation engines and design environment. 2014 12th IEEE International Conference on Solid-State and Integrated Circuit Technology. 
[5] I Delgado, S Skidmore, L Dunleavy. NI AWR Design Environment/Axiem EM co-simulation with Modelithics models. Wireless \& Microwave Technology Conference, 2015:1-4.

[6] TV Dinh, D Lesénéchal, B Domengès. Modeling and characterization of bond-wire arrays for distributed Chip-Package-PCB Co-design. Microwave Conference, 2015, 133:9-19.

[7] S Koziel, A Bekasiewicz. EM-simulation-driven design optimization of compact microwave structures using multi-fidelity simulation models and adjoint sensitivities. International Journal of RF and Microwave Computer-Aided, 2016, 26 (5):442-448.

[8] M Thompson, J Moore. Challenges and methodologies in EM simulation with circuit models. Bipolar/bicmos Circuits \& Technology Meeting-bctm, 2015:20-26.

[9] F Passos, E Roca, R Castro-López, et al. A strategy to efficiently include electromagnetic simulations in optimization-based RF circuit design methodologies. IEEE MTT-S International Conference on Numerical Electromagnetic and Multiphysics Modeling and Optimization for RF, Microwave, and Terahertz Applications (NEMO) 2017:64-66.

[10] Bin Wan, Cindy Zhang, Xingang Wang. Multi-Technology
Simulation with Mixed Design Environments. IEEE International Conference on Asic, 2016:1-4.

[11] Xinqing Sheng. Essentials of Computational Electromagnetics ( $3^{\text {rd }}$ Edition). China, Science Press, 2018: 1-217.

[12] $\mathrm{Hu}$ Laiping and Liu Zhanjun. Comparison Among Computational Methods of Electromagnetics [J]. Modern Electronics Technique, 2003, No. 10: 75-78.

[13] X Dong, T An. A new FEM approach for open boundary Laplace's problem. IEEE Transactions on Microwave Theory and Techniques 44(1):157 - 160 - February 1996.

[14] T Eibert, V Hansen. Calculation of unbounded field problems in free space by a 3D FEM/BEM-hybrid approach. Journal of Electromagnetic Waves \& Applications, 2012, 10 (1):61-78.

[15] Xingfu Xu. ADS2011 Radio Frequency Circuit Design and Simulation Examples. China, Publishing House of Electronics Industry, May 2014: 1-523.

[16] Xingfu Xu. HFSS Radio Frequency Simulation Design Case Studies. China, Publishing House of Electronics Industry, May 2015: 1-583.

[17] Weimin Sun. Accurate EM Simulation of SMT Components in RF Designs. 2017 IEEE Radio Frequency Integrated Circuits Symposium. 